INSTITUT NATIONAL DE RECHERCHE EN INFORMATIQUE ET EN AUTOMATIQUE

\title{
Asymptotics of the Perron eigenvalue and eigenvector using Max-algebra
}

\author{
Marianne Akian, Ravindra Bapat, Stéphane Gaubert
}

No 3450

Juillet 1998

THÈME 4 



\title{
Asymptotics of the Perron eigenvalue and eigenvector using Max-algebra
}

\author{
Marianne Akian* , Ravindra Bapat ${ }^{\dagger}$, Stéphane Gaubert ${ }^{\ddagger}$ \\ Thème 4 - Simulation et optimisation \\ de systèmes complexes \\ Projets Meta2
}

Rapport de recherche $n^{\circ} 3450$ - Juillet 1998 - 12 pages

\begin{abstract}
We consider the asymptotics of the Perron eigenvalue and eigenvector of irreducible nonnegative matrices whose entries have a geometric dependance in a large parameter. The first term of the asymptotic expansion of these spectral elements is solution of a spectral problem in a semifield of jets, which generalizes the max-algebra. We state a "Perron-Frobenius theorem" in this semifield, which allows us to characterize the first term of this expansion in some non-singular cases. The general case involves an aggregation procedure à la Wentzell-Freidlin.
\end{abstract}

Key-words: Perron-Frobenius Theorem, Max-algebra, Perturbation of eigenvalues, Perturbation of linear operators, Asymptotics, Freidlin-Wentzell theory, Large deviations

(Résumé : tsvp)

\footnotetext{
* Email: Marianne.Akian@inria.fr

$\dagger$ Indian Statistical Institute, New Delhi, 110016, India. E-mail: rbb@isid. ernet. in

$\ddagger$ Email: Stephane.Gaubert@inria.fr
} 


\section{Asymptotiques de la valeur propre et du vecteur propre de Perron via l'algèbre max-plus}

Résumé : On s'intéresse à l'asymptotique de la valeur propre et du vecteur propre de Perron de matrices à coefficients positifs ou nuls, dépendant géométriquement d'un grand paramètre. Le premier terme du développement asymptotique de ces élements spectraux est solution d'un problème spectral sur un semi-corps de jets, qui généralise le semi-corps max-plus. Nous établissons un "théorème de PerronFrobenius" pour les jets, qui nous permet de caractériser le premier terme de ce développement dans des cas non-singuliers. Le cas général requiert une procédure d' agrégation à la Wentzell-Freidlin.

Mots-clé : Théorème de Perron-Frobenius, Algèbre max-plus, Perturbation de valeurs propres, Perturbation d'opérateurs linéaires, Asymptotiques, Théorie de Freidlin-Wentzell, Grandes déviations 


\section{Introduction}

Let $\mathcal{A}_{p}$ denote a $n \times n$ nonnegative matrix, depending on a large real parameter $p$. We consider the nonnegative spectral problem:

$$
\mathcal{A}_{p} \mathcal{U}_{p}=\mathcal{L}_{p} \mathcal{U}_{p}, \quad \mathcal{U}_{p} \in\left(\mathbb{R}^{+}\right)^{n} \backslash 0, \quad \mathcal{L}_{p} \in \mathbb{R}^{+},
$$

where $\mathbb{R}^{+}$denotes the set of nonnegative real numbers. When $\mathcal{A}_{p}$ is irreducible, $\mathcal{L}_{p}$ is unique, and it is called the Perron eigenvalue of $\mathcal{A}_{p}$ (see e.g. [4, Ch. 2]). We call normalized Perron eigenvector the unique $\mathcal{U}_{p}$ that satisfies $\sum_{i}\left(\mathcal{U}_{p}\right)_{i}=1$. In this note, we adress the following problem: can we determine the asymptotic behavior of $\mathcal{L}_{p}$ and $\mathcal{U}_{p}$ from that of $\mathcal{A}_{p}$ ?

We begin with an elementary large deviation type result, which extends the result given in [10] for $\mathcal{A}_{p}=\left(A_{i j}^{p}\right)$.

THEOREM 1 (LARGE DEVIATION OF $\mathcal{L}_{p}$ ). If the limits

$$
A_{i j} \stackrel{\text { def }}{=} \lim _{p \rightarrow \infty}\left(\mathcal{A}_{p}\right)_{i j}^{\frac{1}{p}}
$$

exist for $i, j=1, \ldots, n$, and if $A=\left(A_{i j}\right)$ is irreducible, then

$$
\lim _{p \rightarrow \infty}\left(\mathcal{L}_{p}\right)^{\frac{1}{p}}=\max _{1 \leq k \leq n} \max _{i_{1} \ldots i_{k}}\left(A_{i_{1} i_{2}} A_{i_{2} i_{3}} \ldots A_{i_{k} i_{1}}\right)^{\frac{1}{k}} .
$$

Indeed, $0 \leq\left(\mathcal{U}_{p}\right)_{i} \leq \sum_{j}\left(\mathcal{U}_{p}\right)_{j}=1$. Hence, $\left(\mathcal{U}_{p}\right)_{i}^{\frac{1}{p}}$, which is bounded, has a limit point $0 \leq U_{i} \leq 1$, and $\max _{j} U_{j}=1$. It follows from (1) that $\left(\mathcal{L}_{p}\right)^{\frac{1}{p}}$ also has a limit point $\Lambda$, which satisfies

$$
\max _{j} A_{i j} U_{j}=\Lambda U_{i}, \text { for } i=1, \ldots, n
$$

Now, it is convenient to introduce the max-times semifield $\mathbb{R}_{\max }=$ $\left(\mathbb{R}^{+}, \max , \times, 0,1\right)$. We recognize in (4) a spectral problem for the matrix $A$ in

\footnotetext{
${ }^{1}$ A semiring $(S, \oplus, \otimes, 0,1)$ is a set $S$ equipped with two laws $(a, b) \mapsto a \oplus b,(a, b) \mapsto a \otimes b$, called addition and multiplication, respectively, such that $(S, \oplus, 0)$ is a commutative monoid, $(S, \otimes, 1)$ is a monoid, the multiplication distributes over the addition, and the zero element 0 is absorbing for multiplication. A semifield is a semiring whose non zero elements have an inverse. In any semiring, we can defi ne the matrix multiplication as usual (e.g. in $\mathbb{R}_{\max },(A U)_{i}=\bigoplus_{j} A_{i j} \otimes U_{j}=$ $\max _{j} A_{i j} U_{j}$ ).
}

RR n 3450 
the semifield $\mathbb{R}_{\max }$. The $\mathbb{R}_{\max }$ analogue of the Perron-Frobenius theorem states that an irreducible matrix $A$ has a unique eigenvalue, given by the maximal circuit mean $\rho_{\max }(A)$, which, by definition, is the right hand side of (3) (see e.g. [2, Th. 3.100],[6, $\S \mathrm{VI}],[11, \S 3.7])$. Thus, $\Lambda=\rho_{\max }(A)$ holds for all limit points $\Lambda$ of $\left(\mathcal{L}_{p}\right)^{\frac{1}{p}}$. This proves Theorem 1.

The above argument does not guarantee the convergence of $\left(\mathcal{U}_{p}\right)^{\frac{1}{p}}$, except when all the eigenvectors of $A$ are proportional: this simple case is dealt with in $\S 2$. In $\S 3$, we show that if the non-zero entries of $\mathcal{A}_{p}$ have asymptotic expansions of the form

$$
\left(\mathcal{A}_{p}\right)_{i j} \sim a_{i j} A_{i j}^{p}
$$

then $\mathcal{L}_{p}$ has an asymptotic expansion of the same form. This expansion is the unique eigenvalue of $\left(a_{i j} A_{i j}^{p}\right)$, seen as a matrix with entries in a semifield of jets. When all the eigenvectors of the later matrix are proportional, the entries of $\mathcal{U}_{p}$ also have asymptotic expansions of the form (5). However, in general, (5) need not imply the existence of the limits $U_{i} \stackrel{\text { def }}{=} \lim _{p \rightarrow \infty}\left(\mathcal{U}_{p}\right)_{i}^{\frac{1}{p}}$, as shown by the following counter example:

$$
\begin{gathered}
\mathcal{A}_{p}=\left[\begin{array}{cc}
1+\cos (p) e^{-p} & e^{-2 p} \\
e^{-2 p} & 1
\end{array}\right], \\
\liminf _{p \rightarrow \infty}\left(\frac{\left(\mathcal{U}_{p}\right)_{2}}{\left(\mathcal{U}_{p}\right)_{1}}\right)^{\frac{1}{p}}=e^{-1}<\limsup _{p \rightarrow \infty}\left(\frac{\left(\mathcal{U}_{p}\right)_{2}}{\left(\mathcal{U}_{p}\right)_{1}}\right)^{\frac{1}{p}}=e .
\end{gathered}
$$

In [1], we prove via an extension of the Puiseux expansion theorem that when the entries of $\mathcal{A}_{p}$ have Dirichlet series expansions (see [13],[14, Ch. VI]), $\mathcal{L}_{p}$ and the entries of $\mathcal{U}_{p}$ also have Dirichlet series expansions. Then, a fortiori, the limit $U=$ $\left(U_{i}\right)$ exists. It can be computed using an aggregation procedure. In $\S 4$, we only present the first step of this procedure, which is enough to determine $U$ in some non-singular cases.

The problem of computing the limits $\Lambda$ and $U$ arises in particular in Statistical Mechanics, when using the transfer operator methods at small temperatures $T=1 / p$ (see e.g. [3],[5]). Some of the results given below can be seen as partial extensions to the case of nonnegative matrices of the classical Freidlin-Wentzell singular perturbation results $[9$, Ch. 6] which deal with the special case of Markov 
matrices $\mathcal{A}_{p}$. Other max-algebra related (W.K.B. type) asymptotic results have been obtained in [7].

The proofs of the results presented here will be detailed in [1].

\section{When max-times spectral theory determines the asymptotics}

Let $(S, \oplus, \otimes, 0,1)$ denote an arbitrary semiring. With a $n \times n$ matrix $A$ with entries in $S$, we associate (as in conventional Perron-Frobenius theory) a digraph $\mathrm{G}(A)$ with nodes $1, \ldots, n$, and set of $\operatorname{arcs}\left\{(i, j) \mid A_{i j} \neq 0\right\}$. We say that $A$ is irreducible if $\mathrm{G}(A)$ is strongly connected.

When the reflexive and transitive relation $\preceq$, defined by $a \preceq b \Longleftrightarrow \exists c, b=$ $a \oplus c$, is an order relation, and in particular, when $S=\mathbb{R}_{\max }$, we define the Kleene star $a^{*}$ as the least upper bound of the monotone sequence $\left(\bigoplus_{1 \leq k \leq K} a^{k}\right)_{K \geq 1}$, when it exists.

When $S$ is the $\mathbb{R}_{\max }$ semiring, we say that a circuit $c=\left(i_{1}, \ldots, i_{k}\right)$ is critical if its mean geometric weight $\left(A_{i_{1} i_{2}} A_{i_{2} i_{3}} \ldots A_{i_{k} i_{1}}\right)^{\frac{1}{k}}$ attains the maximum in the right hand side of (3). The critical graph $\mathrm{CG}(A)$ is the subgraph of $\mathrm{G}(A)$, composed uniquely of the nodes and arcs in critical circuits. The strongly connected components of the critical graph are called critical classes. We set $\tilde{A}=\rho_{\max }(A)^{-1} A$. Then, $(\tilde{A})^{*}$ exists. A column of $(\tilde{A})^{*}$, whose index lies in a critical class, is called critical. The max-times spectral theorem (see [2, Th. 3.100], [6, Th. VI.10], [11, §3.7] and the references therein) states that if we select (arbitrarily) one critical column per critical class, we obtain a minimal generating set of the eigenspace of an irreducible matrix $A$. As an application of this result, we obtain:

THEOREM 2 (LARGE DEVIATION OF $\mathcal{U}_{p}$ ). If $\mathcal{A}_{p}$ satisfies the assumptions of Theorem 1 , and if $A$ has a unique critical class, then

$$
\lim _{p \rightarrow \infty}\left(\mathcal{U}_{p}\right)_{i}^{\frac{1}{p}}=\frac{(\tilde{A})_{i j}^{*}}{\bigoplus_{k}(\tilde{A})_{k j}^{*}}, \text { for } i=1, \ldots, n
$$

where $j$ is an arbitrary node of this critical class.

$\mathrm{RR} \mathrm{n}^{\circ} 3450$ 
Recall that $(\tilde{A})^{*}$ is equal to $\bigoplus_{0 \leq k \leq n-1}(\tilde{A})^{k}$, and that it can be computed in $O\left(n^{3}\right)$ time using semiring versions of Gauss algorithm (see [2, Th. 3.20] and [12, Ch. 3,Algo. 3], respectively).

\section{When the spectral theory of max-jets determines the asymptotics}

We denote by $\mathbb{J}_{\max }$ the semifield with set of elements $\{(b, B) \quad \mid \quad b>0, B>$ $0\} \cup\{(0,0)\}$, equipped with the two laws

$$
\begin{aligned}
& (b, B) \oplus(c, C)= \begin{cases}(b, B) & \text { if } B>C \\
(c, C) & \text { if } B<C \\
(b+c, B) & \text { if } B=C\end{cases} \\
& (b, B) \otimes(c, C)=(b c, B C) .
\end{aligned}
$$

The zero element $(0,0)$ and the unit $(1,1)$ will be denoted by 0,1 , respectively. This semifield was introduced in [8]. It is isomorphic to the semifield of asymptotic expansions of the form $b B^{p}+o\left(B^{p}\right)$ around $p=\infty$, equiped with the usual addition and multiplication.

We will say that a nonnegative real valued function $f$ of a large parameter $p$ has a first max-jet $(b, B)$, and we will write $f(p) \sim(b, B)$, if $f(p)=b B^{p}+o\left(B^{p}\right)$ around $p=\infty$ (when $(b, B)=0$, this means that $f(p)=0$ for $p$ parge enough). The above definition and notation will be extended to matrices and vectors (entrywise).

If $\mathcal{A}_{p}$ and $\mathcal{U}_{p}$ have first max-jets $\mathcal{A} \in\left(\mathbb{J}_{\max }\right)^{n \times n}$ and $\mathcal{U} \in\left(\mathbb{J}_{\text {max }}\right)^{n}$ respectively, it follows from (1) that $\mathcal{L}_{p}$ has also a first max-jet $\mathcal{L} \in \mathbb{J}_{\text {max }}$, which satisfies $\mathcal{A U}=$ $\mathcal{L} \mathcal{U}$. Thus, the max-jet $\mathcal{U}$ of $\mathcal{U}_{p}$, if it exists, will be characterized in the particular cases when all the eigenvectors of $\mathcal{A}$ are proportional. We next state a $\mathbb{J}_{\max }$ analogue of the Perron-Frobenius theorem.

For any subgraph $C$ of the digraph associated with a matrix $A$ with entries in any semiring, we denote by $A^{\mathrm{C}}$ the matrix with entries $A_{i j}^{\mathrm{C}}=A_{i j}$ if $(i, j)$ is an arc of $\mathrm{C}$, and $A_{i j}^{\mathrm{C}}=0$ otherwise. Given an eigenvector $U \in\left(\mathbb{R}_{\max }\right)^{n}$ of a matrix $A \in\left(\mathbb{R}_{\max }\right)^{n \times n}$, the saturation graph $\mathrm{S}(A, U)$ is the subgraph of $\mathrm{G}(A)$ with set of $\operatorname{arcs}\left\{(i, j) \mid A_{i j} U_{j}=\rho_{\max }(A) U_{i}\right\}$. 
Let $\mathcal{A}=(a, A) \in\left(\mathbb{J}_{\max }\right)^{n \times n}$. Clearly, $\mathcal{A}$ has an eigenvector $\mathcal{U}=(u, U) \in$ $\left(\mathbb{J}_{\max }\right)^{n}$, with eigenvalue $\mathcal{L}=(\lambda, \Lambda)$, iff

$$
A U=\Lambda U, \quad a^{S(A, U)} u=\lambda u
$$

(the first identity is a spectral problem in $\mathbb{R}_{\max }$, the second identity is an ordinary nonnegative spectral problem). The saturation graph in general depends on the particular choice of $U$, but when $A$ is irreducible, for all eigenvectors $U$ of $A$, $\mathrm{CG}(A) \subset \mathrm{S}(A, U)$, and any circuit of $\mathrm{S}(A, U)$ is contained in $\mathrm{CG}(A)$. The matrix $a^{\mathrm{CG}(A)}$ is block diagonal, the blocks being exactly the critical classes. We call basic classes of $\mathcal{A}=(a, A)$ the basic classes of $a^{\mathrm{CG}(A)}$ in the usual sense, i.e. the classes with maximal Perron eigenvalue. We denote by $\rho(b)$ the usual Perron eigenvalue of a matrix $b$.

Theorem 3 ("Perron-Frobenius Theorem” for MAX-Jets). An irreducible matrix $\mathcal{A}=(a, A) \in\left(\mathbb{J}_{\max }\right)^{n \times n}$ admits the unique eigenvalue

$$
\rho_{\mathbb{J}}(\mathcal{A}) \stackrel{\text { def }}{=}\left(\rho\left(a^{\mathrm{CG}(A)}\right), \rho_{\max }(A)\right) .
$$

The characterization of the eigenspace is more subttle in $\mathbb{J}_{\max }$ than in $\mathbb{R}_{\max }$. We will only need the following simple result.

THEOREM 4 (GEOMETRIC SIMPliCITy OF THE EIGENVAlue). An irreducible matrix $\mathcal{A}=(a, A) \in\left(\mathbb{J}_{\max }\right)^{n \times n}$ has a unique eigenvector (up to a proportionality factor) iff it has a unique basic class. An eigenvector $\mathcal{U}=(u, U)$ is obtained as follows: $U$ is a column of $(\tilde{A})^{*}$, whose index belongs to the basic class; $u$ is a positive eigenvector of $a^{\mathrm{S}(A, U)}$.

As a consequence of Theorems 3 and 4, we obtain:

THEOREM 5 (FIRST ORDER ASYMPTOTICS). If $\mathcal{A}_{p}$ has a first max-jet $\mathcal{A} \in$ $\left(\mathbb{J}_{\max }\right)^{n \times n}$, then

$$
\mathcal{L}_{p} \sim \rho_{\mathbb{J}}(\mathcal{A})
$$

Moreover, if $\mathcal{A}$ has a unique basic class, then $\mathcal{U}_{p}$ has a first max-jet, which is the unique eigenvector $\mathcal{U}$ of $\mathcal{A}$ with sum 1.

$\mathrm{RR} \mathrm{n}^{\circ} 3450$ 


\section{Aggregated matrix}

When the matrix $\mathcal{A}$ has several basic classes, the determination of the limit eigenvector relies on an aggregation procedure, the first step of which we next describe.

We denote by $\mathrm{B}_{1}, \ldots, \mathrm{B}_{s}$ the basic classes of $\mathcal{A}$. We set $\mathrm{B}=\cup_{1 \leq i \leq s} \mathrm{~B}_{i}$ and $\mathrm{N}=\{1, \ldots, n\} \backslash \mathrm{B}$. Let $\mathcal{V}_{1}, \ldots, \mathcal{V}_{s}$ be eigenvectors of the submatrices $\mathcal{A}_{\mathrm{B}_{1} \mathrm{~B}_{1}}, \ldots, \mathcal{A}_{\mathrm{B}_{s} \mathrm{~B}_{s}}$, respectively (for all subsets $J, K \subset\{1, \ldots, n\}, \mathcal{A}_{J K}$ denotes the $J \times K$ submatrix of $\mathcal{A}$ ). The following key lemma is a consequence of the fact that $a u \leq \rho(a) u$ implies $a u=\rho(a) u$, for all irreducible nonnegative matrices $a$ and nonnegative vectors $u$ (see e.g. [4, Ch. 1,Th. 3.35]).

LEMMA 6. Any eigenvector $\mathcal{U}$ of an irreducible matrix $\mathcal{A} \in\left(\mathbb{J}_{\max }\right)^{n \times n}$ is of the form $\mathcal{U}=\mathcal{V} \mathcal{U}^{\prime}$, for some $\mathcal{U}^{\prime} \in\left(\mathbb{J}_{\max }\right)^{s}$, where

$$
\mathcal{V} \stackrel{\text { def }}{=}\left[\begin{array}{c}
I \\
\left(\rho_{\mathbb{J}}(\mathcal{A})^{-1} \mathcal{A}_{\mathrm{NN}}\right)^{*} \rho_{\mathbb{J}}(\mathcal{A})^{-1} \mathcal{A}_{\mathrm{NB}}
\end{array}\right] \operatorname{diag}\left(\mathcal{V}_{1}, \ldots, \mathcal{V}_{s}\right)
$$

Here, $I$ is the $\mathrm{B} \times \mathrm{B}$ identity matrix, and for all (possibly rectangular) matrices $X_{1}, \ldots, X_{k}, \operatorname{diag}\left(X_{1}, \ldots, X_{k}\right)$ denotes the (possibly rectangular) block diagonal matrix with diagonal blocks $X_{1}, \ldots, X_{k}$.

In the sequel, we will identify the max-jet (resp. the matrix of max-jets) $(b, B)$ to the function $p \mapsto b B^{p}$ (resp. $p \mapsto\left(b_{i j} B_{i j}^{p}\right)$ ). This allows us to write $\mathcal{A}_{p}=$ $\mathcal{A}^{\mathrm{BG}(\mathcal{A})}+\mathcal{R}_{p}$, where $\mathrm{BG}(\mathcal{A})$ denotes the subgraph of $\mathrm{CG}(\mathcal{A})$ with set of nodes $\mathrm{B}$. In general, the remainder matrix $\mathcal{R}_{p}$ has negative entries.

Let $\mathcal{M}_{1}, \ldots, \mathcal{M}_{s}$ denote the left eigenvectors of the submatrices $\mathcal{A}_{\mathrm{B}_{1} \mathrm{~B}_{1}}, \ldots, \mathcal{A}_{\mathrm{B}_{s} \mathrm{~B}_{s}}$, respectively, normalized by the condition $\mathcal{M}_{i} \mathcal{V}_{i}=1$, for $i=1, \ldots, s$. We set $\mathcal{M}=\operatorname{diag}\left(\mathcal{M}_{1}, \ldots, \mathcal{M}_{s}\right)\left[\begin{array}{ll}I & 0\end{array}\right](0$ is the $\mathrm{B} \times \mathrm{N}$ zero matrix). Left multiplying $\mathcal{A}_{p} \mathcal{U}_{p}=\mathcal{L}_{p} \mathcal{U}_{p}$ by $\mathcal{M}$, we obtain

$$
\mathcal{M R}_{p} \mathcal{U}_{p}=\left(\mathcal{L}_{p}-\rho_{\mathbb{J}}(\mathcal{A})\right) \mathcal{M U}_{p}
$$

Using Lemma 6, we obtain the following result.

THEOREM 7 (SECOND ORDER ASYMPTOTICS). If $\mathcal{A}_{p}$ and $\mathcal{R}_{p}$ have first max-jets $\mathcal{A}$ and $\mathcal{R}$, respectively, then

$$
\mathcal{L}_{p}=\rho_{\mathbb{J}}(\mathcal{A})+\rho_{\mathbb{J}}\left(\mathcal{A}^{\prime}\right)+o\left(\rho_{\mathbb{J}}\left(\mathcal{A}^{\prime}\right)\right),
$$

INRIA 
where $\mathcal{A}^{\prime} \stackrel{\text { def }}{=} \mathcal{M R V} \in\left(\mathbb{J}_{\max }\right)^{s \times s}$. Moreover, if $\mathcal{A}^{\prime}$ has a unique basic class, then $\mathcal{U}_{p}$ has a first max-jet, which is the unique vector with sum 1 of the form $\mathcal{V} \mathcal{U}^{\prime},<\mathcal{U}^{\prime}$ being an eigenvector of $\mathcal{A}^{\prime}$, and $\mathcal{V}$ being defined in (10).

Example 8. Consider the transfer matrix of the simplest one dimensional Ising model [3, Ch. 2]

$$
\mathcal{A}_{1 / T}=\left[\begin{array}{cc}
\exp ((J+H) / T) & \exp (-J / T) \\
\exp (-J / T) & \exp ((J-H) / T)
\end{array}\right], \text { with } J>0, H \in \mathbb{R} .
$$

Setting $K=\exp (J)>1, L=\exp (H)>0, p=1 / T$, we can write the first max-jet of $\mathcal{A}_{p}$ as $\mathcal{A}=(a, A)$ with $a=\left[\begin{array}{ll}1 & 1 \\ 1 & 1\end{array}\right]$ and $A=\left[\begin{array}{cc}K L & K^{-1} \\ K^{-1} & K L^{-1}\end{array}\right]$. We have $\rho_{\mathbb{J}}(\mathcal{A})=\left(1, \max \left(K L, K L^{-1}\right)\right)$. Thus, $\mathcal{L}_{p} \sim\left(\max \left(K L, K L^{-1}\right)\right)^{p}$. When $H>0$, there is a unique critical class, $C_{1}=\{1\}$, and $\tilde{A}=\left[\begin{array}{c}{ }^{1} L^{-1} \\ K^{-2} L^{-1}\end{array} L^{-2}\right],(\tilde{A})^{*}=$ $\left[{ }_{K^{-2} L^{-1}}^{1} K^{-2} L^{-1}\right]$. By Theorem 5, $\mathcal{U}_{p} \sim\left[1\left(K^{-2} L^{-1}\right)^{p}\right]^{T}$. By symmetry, if $H<0$, then $\mathcal{U}_{p} \sim\left[\left(K^{-2} L^{-1}\right)^{p} 1\right]^{T}$ : the limit eigenvector at zero temperature is selected by the sign of the external magnetic field $H$. When $H=0, \mathcal{A}$ has two distinct critical classes $C_{1}=\{1\}, C_{2}=\{2\}$, that are both basic. Theorem 7 allows us to determine the equivalent of $\mathcal{U}_{p}$. Indeed, $\mathcal{V}=\mathcal{M}=I$ (the $2 \times 2$ identity matrix), and $\mathcal{A}^{\mathrm{BG}(\mathcal{A})}=\left[\begin{array}{cc}(1, K) & 0 \\ 0 & (1, K)\end{array}\right]$. We obtain $\mathcal{R}_{p}=\mathcal{R}=\mathcal{A}^{\prime}=\left[\begin{array}{cc}0 & { }^{\left(1, K^{-1}\right)} \\ \left(1, K^{-1}\right) & 0\end{array}\right]$. Thus, $\rho_{\mathbb{J}}\left(\mathcal{A}^{\prime}\right)=\left(1, K^{-1}\right), \mathcal{L}_{p}=K^{p}+K^{-p}+o\left(K^{-p}\right)$, and $\mathcal{U}_{p} \sim\left[\begin{array}{cc}\frac{1}{2} & \frac{1}{2}\end{array}\right]^{T}$.

\section{Version française abrégée}

Soit $\mathcal{A}_{p}$ une matrice $n \times n$ à coefficients réels positifs ou nuls, définie au voisinage de $p=+\infty$. On considère le problème spectral (1) dans le cas où $\mathcal{A}_{p}$ est irréductible : $\mathcal{L}_{p}$ est unique et il existe un unique $\mathcal{U}_{p}$ vérifiant $\sum_{i}\left(\mathcal{U}_{p}\right)_{i}=1$ (voir par exemple [4, Ch. 2]). On cherche à déterminer les asymptotiques de $\mathcal{L}_{p}$ et $\mathcal{U}_{p}$ à partir de celles de $\mathcal{A}_{p}$.

En utilisant les résultats analogues au théorème de Perron-Frobenius dans le semi-corps $\mathbb{R}_{\max }=\left(\mathbb{R}^{+}, \max , \times, 0,1\right)$, isomorphe au semi-corps max-plus (voir par exemple [2, Th. 3.100],[6, §VI],[11, §3.7]), on obtient les asymptotiques de type grandes déviations de $\mathcal{L}_{p}$, et dans certains cas celles de $\mathcal{U}_{p}$. 
ThÉORÈme 1. Si les limites (2) existent et si $A=\left(A_{i j}\right)$ est irréductible, alors $\lim _{p \rightarrow \infty}\left(\mathcal{L}_{p}\right)^{\frac{1}{p}}$ existe. Elle est égale à la valeur propre de $A$ dans $\mathbb{R}_{\max }$, notée $\rho_{\max }(A)$, donnée par le second membre de (3).

Un circuit $c=\left(i_{1}, \ldots, i_{k}\right)$ est dit critique si $\left(A_{i_{1} i_{2}} A_{i_{2} i_{3}} \ldots A_{i_{k} i_{1}}\right)^{\frac{1}{k}}$ réalise le maximum dans (3). On appelle graphe critique le graphe orienté formé des nouds et arcs des circuits critiques. On appelle classe critique une composante fortement connexe du graphe critique. On pose $\tilde{A}=\rho_{\max }(A)^{-1} A$, et on note $(\tilde{A})^{*}=\bigoplus_{k=0}^{\infty}(\tilde{A})^{k}$ l'étoile de Kleene de $\tilde{A}$ (la somme et les puissances sont dans $\mathbb{R}_{\text {max }}$ ).

THÉORÈmE 2. Si $\mathcal{A}_{p}$ satisfait aux hypothèses du théorème 1, et si A n'a qu'une classe critique, alors $\lim _{p \rightarrow \infty}\left(\mathcal{U}_{p}\right)_{i}^{\frac{1}{p}}=U_{i}$ où $U=\left(U_{i}\right)$ est l'unique vecteur propre de $A$ dans $\mathbb{R}_{\max }$ vérifiant $\max _{i} U_{i}=1$. Celui-ci est proportionnel à n'importe quelle colonne de $(\tilde{A})^{*}$ d'indice critique.

Afin d'obtenir des asymptotiques plus précises, on utilise le semi-corps de jets $\mathbb{J}_{\max }$ (introduit dans [8]) composé de l'ensemble des couples $(b, B)$, avec $b, B>0$ ou $b=B=0$, muni des lois (6). On dit que la fonction $f$ de $p$ admet un premier max-jet si $f(p)=b B^{p}+o\left(B^{p}\right)$ autour de $p=+\infty$. On note alors $f(p) \sim(b, B)$. On étend cette notation aux vecteurs et matrices (coordonnée par coordonnée).

ThÉORÈme 3. Si $\mathcal{A}_{p} \sim \mathcal{A}=(a, A) \in\left(\mathbb{J}_{\max }\right)^{n \times n}$, avec $\mathcal{A}$ irréductible, alors $\mathcal{L}_{p} \sim \rho_{\mathbb{J}}(\mathcal{A})$ où $\rho_{\mathbb{J}}(\mathcal{A})=\left(\rho\left(a^{\mathrm{CG}(A)}\right), \rho_{\max }(A)\right)$ est la valeur propre de $\mathcal{A}$ dans $\mathbb{J}_{\max }, a^{\mathrm{CG}(A)}$ est la matrice obtenue à partir de a en annulant les coefficients $a_{i j}$ tels que l'arc $(i, j) n$ 'est pas dans le graphe critique, et où $\rho(\cdot)$ désigne la valeur propre de Perron.

Si a ${ }^{\mathrm{CG}(A)} n^{\prime}$ 'a qu'une classe basique, alors $\mathcal{U}_{p} \sim \mathcal{U}$, l'unique vecteur propre de $\mathcal{A}$ dans $\mathbb{J}_{\max }$ de somme 1 . Celui-ci est de la forme $(u, U)$, où $U$ est une colonne de $(\tilde{A})^{*}$ d'indice basique et $u$ est un vecteur propre positif de la matrice a ${ }^{\mathrm{S}(A, U)}$, obtenue en annulant les $a_{i j}$ tels que $A_{i j} U_{j}<\rho_{\max }(A) U_{i}$.

On appellera classes basiques de $\mathcal{A}$ les classes basiques de $a^{\mathrm{CG}(A)}$. Si $\mathcal{A}$ admet les classes basiques $\mathrm{B}_{1}, \ldots, \mathrm{B}_{s}$, alors tout vecteur propre $\mathcal{U}$ de $\mathcal{A}$ dans $\mathbb{J}_{\max }$ s'écrit $\mathcal{U}=\mathcal{V} \mathcal{U}^{\prime}$, où $\mathcal{V}$ est donné par (10). Dans (10), $\mathcal{V}_{1}, \ldots, \mathcal{V}_{s}$ sont des vecteurs propres de $\mathcal{A}_{\mathrm{B}_{1} \mathrm{~B}_{1}}, \ldots, \mathcal{A}_{\mathrm{B}_{s} \mathrm{~B}_{s}}$, respectivement, $\mathcal{A}_{J K}$ désigne la $J \times K$ sous-matrice de $\mathcal{A}$, 
$\mathrm{B}=\cup_{1 \leq i \leq s} \mathrm{~B}_{i}, \mathrm{~N}=\{1, \ldots, n\} \backslash \mathrm{B}$, et l'étoile dans $\mathbb{J}_{\max }$ est définie par la même formule que dans $\mathbb{R}_{\max }$. Soit $\mathcal{M}=\operatorname{diag}\left(\mathcal{M}_{1}, \ldots, \mathcal{M}_{s}\right)\left[\begin{array}{ll}I & 0\end{array}\right]$, où $\mathcal{M}_{1}, \ldots, \mathcal{M}_{s}$ désignent les vecteurs propres à gauche de $\mathcal{A}_{\mathrm{B}_{1} \mathrm{~B}_{1}}, \ldots, \mathcal{A}_{\mathrm{B}_{s} \mathrm{~B}_{s}}$, respectivement, vérifiant $\mathcal{M}_{i} \mathcal{V}_{i}=1$

THÉORÈme 4. Si $\mathcal{A}_{p} \sim \mathcal{A} \in\left(\mathbb{J}_{\max }\right)^{n \times n}$ et $\mathcal{R}_{p}=\mathcal{A}_{p}-\mathcal{A} \sim \mathcal{R} \in\left(\mathbb{J}_{\max }\right)^{n \times n}$, alors $\mathcal{L}_{p}$ admet le développement asymptotique (11), où $\mathcal{A}^{\prime}=\mathcal{M R} \mathcal{R} \in\left(\mathbb{J}_{\max }\right)^{s \times s}$.

Si $\mathcal{A}^{\prime}$ n'a qu'une classe basique, alors $\mathcal{U}_{p} \sim \mathcal{U}$, l'unique élément de $\left(\mathbb{J}_{\max }\right)^{n}$ de somme 1 , de la forme $\mathcal{V} \mathcal{U}^{\prime}$, où $\mathcal{U}^{\prime}$ est un vecteur propre de $\mathcal{A}^{\prime}$, et $\mathcal{V}$ est donné par (10).

\section{References}

[1] M. Akian, R.B. Bapat, and S. Gaubert. Asymptotics of the Perron eigenvalue and eigenvector. In preparation, 1998.

[2] F. Baccelli, G. Cohen, G.J. Olsder, and J.P. Quadrat. Synchronization and Linearity. Wiley, 1992.

[3] R.J. Baxter. Exactly solved models in Statistical Mechanics. Academic Press, New York, 1982.

[4] A. Berman and R.J. Plemmons. Nonnegative matrices in the mathematical sciences. Academic Press, 1979.

[5] W. Chou and R.B. Griffiths. Ground states of one dimensional systems using effective potentials. Phys. Rev. B, 34:6219-34, 1986.

[6] R.A Cuninghame-Green. Minimax algebra and applications. Advances in Imaging and Electron Physics, 90, 1995.

[7] S.Yu. Dobrokhotov, V.N. Kolokoltsov, and V.P. Maslov. Quantization of the Bellman equation, exponential asymptotics and tunneling. In V.P. Maslov and S.N. Samborskiŭ, editors, Idempotent analysis, volume 13 of Adv. in Sov. Math. AMS, RI, 1992.

[8] A.V. Finkelstein and M.A. Roytberg. Computation of biopolymers: a general approach to different problems. BioSystems, 30:1-20, 1993.

RR $n^{\circ} 3450$ 
[9] M.I. Freidlin and A.D. Wentzell. Random Perturbations of Dynamical Systems. Springer, 1984.

[10] S. Friedland. Limit eigenvalues of nonnegatives matrices. Linear Alg. and Appl., 74:173-178, 1986.

[11] S. Gaubert and M. Plus. Methods and applications of $(\max ,+)$ linear algebra. In R. Reischuk and M. Morvan, editors, STACS'97, number 1200 in LNCS, Lübeck, March 1997. Springer.

[12] M. Gondran and M. Minoux. Graphes et algorithmes. Eyrolles, Paris, 1979. Engl. transl. Graphs and Algorithms, Wiley, 1984.

[13] G.H. Hardy and M. Riesz. The general theory of Dirichlet's series. Cambridge University Press, 1915.

[14] J.P. Serre. Cours d'arithmétique. PUF, 1977. 


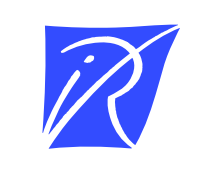

Unit'e de recherche INRIA Lorraine, Technopôle de Nancy-Brabois, Campus scientifi que, 615 rue du Jardin Botanique, BP 101, 54600 VILLERS LÈS NANCY

Unit'e de recherche INRIA Rennes, Irisa, Campus universitaire de Beaulieu, 35042 RENNES Cedex

Unit'e de recherche INRIA Rhône-Alpes, 655, avenue de l'Europe, 38330 MONTBONNOT ST MARTIN

Unit'e de recherche INRIA Rocquencourt, Domaine de Voluceau, Rocquencourt, BP 105, 78153 LE CHESNAY Cedex

Unit ${ }^{\prime}$ de recherche INRIA Sophia-Antipolis, 2004 route des Lucioles, BP 93, 06902 SOPHIA-ANTIPOLIS Cedex

Éditeur

INRIA, Domaine de Voluceau, Rocquencourt, BP 105, 78153 LE CHESNAY Cedex (France)

http://www.inria.fr

ISSN 0249-6399 